\title{
Erratum to: Applications of Quantum Dynamics in Chemistry
}

Fabien Gatti, Benjamin Lasorne, Hans-Dieter Meyer and André Nauts

\section{Erratum to:}

F. Gatti et al., Applications of Quantum Dynamics

in Chemistry, Lecture Notes in Chemistry 98, https://doi.org/10.1007/978-3-319-53923-2

In the original version of the book, the second affiliation of the author André Nauts was missing. His second affiliation "Laboratoire de Chimie Physique (UMR8000), Université de Paris-Sud, Orsay, Essonne, France” has now been inserted. 